\title{
Proximal, Microbiological and Nutritional Characterization in Chinese Potato Flour of the White Variety Colocasia esculenta for Application in Functional Foods
}

VII International Congress of Science, Technology, Entrepreneurship and Innovation (SECTEI 2020)

Corresponding Author:

F. López

franklindamian96@gmail.com

Published: 26 August 2021

Production and Hosting by Knowledge $E$

(c) F. López et al. This article is distributed under the terms of the Creative Commons Attribution License, which permits unrestricted use and redistribution provided that the original author and source are credited.
S OPEN ACCESS

\section{Caracterización Proximal, Microbiológica y Nutricional en Harina de Papa China de la Variedad Blanca Colocasia esculenta Para su Aplicación en Alimentos Funcionales}

\author{
F. López, L. Arboleda, and V. González
}

Facultad de Ciencias Pecuarias, Escuela de Ingeniería Industrias Pecuarias Escuela Superior Politécnica de Chimborazo, Riobamba, Ecuador

\section{Abstract}

The study presents a qualitative-quantitative scope through descriptive statistics for the variables studied with its analyses, with the objective of performing physical, proximal, microbiological and contained characterization of amino acids in Chinese potato flour of the white variety Colocasia esculenta for its application in functional foods. By obtaining the flour, the proximal percentages are evaluated; moisture $9.01 \%$, ash $2.24 \pm 0.15 \%$ protein $2.02 \pm$ $0.02 \%$ fiber $4.25 \pm 0.26 \%$ and fat $0.43 \pm 0.15 \%$. Additionally, the physical-chemical analyses carried out on the flour obtain a pH of $6.66 \pm 0.10 \%$ acidity (oxalic) $2.24 \pm 0.00 \%$ Brix degrees $2 \pm 0.50 \%$. The nutritional values reported in the Chinese potato flour of the white variety lies mainly in its content of Methionine with a value of $21.15 \%$, Alanine with $10.43 \%$, Isoleucine with $8.08 \%$, Threonine with $7.41 \%$, Histidine with $3.65 \%$ and lysine with $3.10 \%$. Finally, the microbiological analysis of the product is determined, concluding that they are within the parameters established in wheat flours with the INEN 616: 2006 standard; thus it is considered of good quality, suitable for human consumption and consequently, for use in the food industry. Therefore, it can be used as functional food or be transformed through the use of different techniques, thus being an important plant genetic resource to improve the nutritional quality of food, productivity, food health, as well as the protection and prevention of diseases in areas of the equator with high levels of malnutrition.

Keywords: characterization, nutritional food, Chinese potato, industrialization, flour.

\section{Resumen}

El estudio presenta un alcance cualitativo-cuantitativo mediante la estadística descriptiva para las variables estudiadas con sus respectivos análisis y el objetivo de realizar la caracterización física, proximal, microbiológica y contenida de aminoácidos en harina de papa China de la variedad blanca Colocasia Esculenta para su aplicación en alimentos funcionales. Mediante la obtención de la harina se evalúa los porcentajes proximales; humedad 9,01\%, cenizas $2,24 \pm 0,15 \%$ proteína $2,02 \pm 0,02 \%$ fibra $4,25 \pm 0,26 \%$ y grasa $0,43 \pm 0.15 \%$. Además, los análisis físico-químicos realizados en la harina se obtiene un $\mathrm{pH}$ de 6,66 $\pm 0,10 \%$ acidez (oxálico) 2,24 $\pm 0,00 \%$ grados Brix $2 \pm 0,50 \%$. Los valores nutricionales reportados en la harina de papa China de la variedad blanca radica principalmente en su contenido de Metionina 
con un valor de $21,15 \%$, Alanina $10,43 \%$, Isoleucina $8,08 \%$, Treonina $7,41 \%$, Histidina $3,65 \%$ y lisina con el $3,10 \%$. Finalmente, se determina el análisis microbiológico del producto concluyendo que se encuentran dentro de los parámetros establecidos en harinas de trigo con la norma INEN 616:2006 de este modo se considera de buena calidad, apta para el consumo humano y consecutivamente empleada en industria alimentaria. Por lo que, puede ser utilizado como alimento funcional o ser trasformado mediante la utilización de distintas técnicas siendo así un importante recurso fitogenético para mejorar la calidad nutricional de los alimentos, la productividad, la sanidad alimentaria, así como la protección y prevención de enfermedades en zonas del ecuador con altos niveles de desnutrición.

Palabras Clave: caracterización, alimento nutricional, papa China, industrialización, harina.

\section{Introducción}

La papa China (Colocasia esculenta L.) perteneciente a la familia de las Aráceas, es uno de los primeros cultivos utilizados por el hombre [1]. Esta especie de origen asiático reconocida al sureste de Asia, entre India e Indonesia es considerada una de las especies de tubérculos con gran potencial en las zonas tropicales del Ecuador ocupando una superficie total de 419 hectáreas [2].También conocida como taro este tubérculo es propio de las zonas tropicales y subtropicales; en la Amazonía ecuatoriana en los últimos años se ha observado un incremento del 10\% anual de la superficie debido al interés del mercado centroamericano, los principales cultivos de papa China se encuentran en la provincia de Pastaza [3]. Sin embargo, la vida útil de la papa China es larga en comparación a otros tubérculos y para una mejor conservación se recomienda condiciones de humedad relativa alta con temperaturas bajas siendo almacenadas a $11-13^{\circ} \mathrm{C}$ y con el $85-90 \%$ de humedad relativa [3].

Si bien es cierto, el gigantesco crecimiento de la población y la alta demanda de alimentos causan un rápido incremento en su costo lo que ha llevado a la búsqueda de fuentes alternativas de energía calórica con aportes nutricionales a precios más económicos para la alimentación y la dieta en la salud humana [4]. Y este tipo de tubérculos son reconocidos como una fuente barata de carbohidratos en relación a los cereales o leguminosas [5].

Por tal motivo, se ha desarrollado la presente investigación con el objetivo de realizar la caracterización física, proximal, microbiológica y contenido de aminoácidos en harina de papa China de la variedad blanca $C$. esculenta, promoviendo así el uso de la harina no muy conocidos en nuestro medio y poco utilizado en la industria de la alimentación los cual brindan grandes beneficios para nuestra alimentación diaria [6]. Por lo que, puede ser utilizado como alimento funcional o ser trasformado mediante la utilización de 
distintas técnicas; aumentando y fortificando la concentración de distintos componentes que se encuentran presentes en forma natural, o adicionado nutrientes esenciales como vitaminas, minerales, proteínas, aminoácidos esenciales o ácidos grasos para resolver deficiencias de la alimentación que se traducen en fenómenos de carencia colectiva mejorando así la calidad nutricional para las personas que lo consumen [7].

En relación a esto, diversos estudios han demostrado que este tubérculo es rico en carbohidratos, sales minerales, vitaminas y ciertos aminoácidos esenciales permitiendo que sea un alimento de un alto valor nutritivo, excelente digestibilidad y el estar disponible todo el año ayuda a contribuir con la creación de ciertos alimentos funcionales para el consumo humano favoreciendo la salud y previniendo distintos tipos de enfermedades [8]. Por tanto, dentro de los procesos de industrialización de este tubérculo se encuentra la harina de papa China que posee varias características físicas y químicas que la hacen especial, tiene compuestos nutricionales muy importantes que son; proteína, grasa, carbohidratos, fibra, calcio, fósforo, elementos muy utilizados por múltiples industrias [9]. Además, la harina de papa China por su deficiencia en gluten, lo hace consumible para las personas celiaquías o intolerantes al gluten, y el consumo de sus fibras naturales ayudan a la limpieza del tracto digestivo reduciendo así los problemas de estreñimiento [10].

Por consiguiente, la importancia de estos recursos fitogenéticos se enfoca también en ley orgánica del régimen de la soberanía alimentaria ecuatoriana que establece según el Art. 9 que el estado asegurará y desarrollará la investigación científica y tecnológica en materia agroalimentaria, que tendrá por objeto mejorar la calidad nutricional de los alimentos, la productividad, la sanidad alimentaria, así como proteger y enriquecer la agrobiodiversidad [11], siendo los patrones principales los pequeños agricultores que se encuentran aliados a la seguridad alimentaria y actores protagónicos en el esfuerzo de los países por lograr un futuro sin hambre.

En otras palabras, la agroindustrialización de este tubérculo presenta valiosas oportunidades y beneficios para los países en desarrollo, en términos de procesos globales de industrialización, incremento económico, rendimiento de las exportaciones, inocuidad y calidad alimentarias [12]. De igual importancia, la harina de papa China por su alto contenido de elementos nutricionales, es especialmente importante en países en vías de desarrollo donde son una fuente de calorías de bajo costo y de ingresos para los pequeños productores, además son componentes clave en el establecimiento de microempresas relacionadas con la agroindustria.

Por otra parte, las aráceas han sido reconocidas por la FAO, organización que ha publicado varios documentos sobre la importancia de estos y otros tubérculos con su contribución a la seguridad alimentaria de los países en desarrollo, por ejemplo, en Centroamérica se producen y exportan cantidades considerables de papa China, siendo Costa Rica uno de los exportadores más importantes a nivel mundial [13]. Además, 
desde el punto de vista nutricional la proteína presente en los diferentes tipos de harinas es un macronutriente importante en los alimentos. Y el interés de la proteína presente en la dieta humana se debe a su capacidad del aporte de aminoácidos para atender al mantenimiento del peso corporal y al incremento de esta durante el crecimiento. Por lo tanto, la limitación en el aporte de energía y de proteína conduce a un retraso en el crecimiento [7].

En general, la papa China en el Ecuador no es consumida ni comercializada de forma significativa, pues la escasa producción se destina al consumo de los anamales debido a la falta de información sobre su uso por tal razón el presente trabajo tiene como objetivo evaluar los cambios proximales en el proceso de deshidratación de la papa China como tubérculo en harina y realizar el análisis nutricional de la harina a partir de papa China de la variedad blanca $C$. esculenta. En resumen, los consumidores Latinoamericanos buscan diversas características y alternativas en los productos de origen vegetal, como que sean elaborados con materias primas regionales ya que pueden ser muy interesantes desde el punto de vista nutricional y sensorial, además de agregar valor a las materias primas y productos de la zona [14].

\section{Metodología}

El actual estudio presenta un alcance cualitativo-cuantitativo mediante la estadística descriptiva para las variables estudiadas puesto que se evaluaron los datos de cada muestra del experimento con su respectivo análisis del porcentaje de humedad para el vegetal fresco y siguiendo el mismo patrón de análisis bromatológico, microbiológico y nutricional, para la harina de papa China en el cual se explica las razones porque se dieron tales resultados, obteniendo de esta manera nuevos conocimientos que aporten a investigaciones futuras.

El proceso experimental se lleva a cabo en el laboratorio de bromatología de la Facultad de Ciencias Pecuarias (FCP), de la Escuela Superior Politécnica de Chimborazo $(\mathrm{ESPOCH})$. Ubicada en el Cantón Riobamba, provincia de Chimborazo a una altitud de 2850 m.s.n. con una temperatura promedio de $22^{\circ} \mathrm{C}$ y una humedad relativa promedio de $60,9 \%$. La papa China (C. esculenta) es procedente de la plaza 5 de Junio del Cantón Baños de la Provincia de Tungurahua, el cual es trasladado al laboratorio de reproducción animal para sus análisis.

\subsection{Variable Dependiente Evaluada en el Vegetal Fresco}

Mediante un muestreo se selecciona los mejores ejemplares de la papa China para ser lavadas con abundante agua y retirar la tierra y materias extrañas. Y con la ayuda de un bisturí se elimina la corteza del tubérculo, luego se realiza un escaldado para evitar 
la oxidación de la papa China para proceder a realizar el análisis proximal de la papa China.

\subsubsection{Determinación de humedad en el vegetal fresco}

Se utiliza $1 \mathrm{~g}$ de muestra previamente realizando el demuestre directamente con el crisol tarado, repartiendo uniformemente la muestra en su base. Se coloca en la estufa a $103 \pm 3^{\circ} \mathrm{C}$ por un lapso de 2 a $3 \mathrm{hr}$, hasta su peso constante. Después se lo enfría en un desecador hasta la temperatura ambiente y se procede a pesar. La determinación del porcentaje de humedad se realiza por duplicado y con la siguiente fórmula.

$$
\begin{gathered}
s s(\%)=\frac{(m 2-m)}{(m 1-m)} \times 100 \\
\% \text { Humedad }=100-\% s s,[15]
\end{gathered}
$$

en donde:

ss: sustancia seca en porcentaje en masa; m: masa de la cápsula en g;

m1: masa de la cápsula con la muestra en g; y

m2: masa de la cápsula con la muestra después del calentamiento en g.

\subsection{Variables Dependientes Evaluadas en la Harina de Papa China}

Un vez retirada la corteza del vegetal fresco se realiza un corte en rebanadas de aproximadamente $1 \mathrm{~mm}$ de grosor y se las coloca sobre rejillas cubiertas con papel aluminio para introducirlas en una estufa memmert a $60^{\circ} \mathrm{C}$ durante un tiempo aproximado de 6 hr y de este modo poder retirar toda la cantidad de humedad de la papa China. Una vez deshidratada las muestras se realiza la molienda utilizando un molino industrial para obtener la granulometría ideal y se procede a almacenar en recipientes herméticos para su posterior análisis. Para realizar los cálculos de cada variable analizada se utiliza el programa operativo Excel.

\subsubsection{Determinación de proteína en el vegetal fresco y la harina de papa China}

Se emplea el método Kjeldahl, para el mismo se utiliza $1 \mathrm{~g}$ de muestra previamente tarado con papel, $9 \mathrm{~g}$ de Na2SO4, $1 \mathrm{~g}$ CuSO 4 se introduce las muestras en los balones de Kjeldahl de $800 \mathrm{ml}$ añadiendo $25 \mathrm{ml}$ de H2SO 4 concentrado, esta mezcla se somete a ebullición hasta obtener un líquido verde esmeralda. Transcurrido ese tiempo se enfría y se coloca $200 \mathrm{ml}$ de $\mathrm{H} 2 \mathrm{O}$ destilada para disolver el contenido que al enfriarse se solidifica, luego se adiciona perlas de ebullición (Zn) más 100 ml de NaOH y se destila, 
posteriormente se colecta en $100 \mathrm{ml}$ de ácido bórico 2,5\% más dos gotas de indicador y se titula con $\mathrm{HCl} 0.1 \mathrm{~N} / 10$ hasta que se torne una coloración rosa.

La determinación del contenido de proteína se realiza por duplicado y debe calcularse aplicando la formula siguiente:

$$
\% P B=\frac{(\mathrm{N} \mathrm{HCl} \mathrm{V} \mathrm{HCl} \times 6.25 \times 0.014)}{W M} \times 100[16]
$$

\subsubsection{Determinación de cenizas en el vegetal fresco y la harina de papa China}

Se utiliza $1 \mathrm{~g}$ de muestra obtenida en el análisis del porcentaje de humedad colocándola en un crisol previamente tarado y se calcina en una plancha calcinadora, posteriormente el crisol más la muestra se somete a calcinación en una mufla Lindberg Blue $\mathrm{M}$ a $550^{\circ} \mathrm{C}$ durante $4 \mathrm{hr}$, la muestra se debe enfriar en un desecador durante $1 \mathrm{hr}$ y pesar en una balanza analítica Pioner TM el crisol más la muestra. Para el cálculo de cenizas se utiliza la siguiente formula.

$$
\% \text { de ceniza } \frac{\text { (peso del crisil + ceniza) }-(\text { peso del crisol) }}{(\text { peso del crisol + muestra) }-(\text { peso del crisol) }} \times 100[17] \text {. }
$$

\subsubsection{Determinación de fibra en el vegetal fresco y la harina de papa China}

Se emplea el método Ankom, para el mismo se pesa $1 \mathrm{~g}$ de muestra y se coloca en el vaso de Berzellius con $100 \mathrm{ml}$ de ácido sulfúrico más $3 \mathrm{ml}$ de alcohol amílico al 7\%, se coloca el vaso en el equipo ajustando al condensador, se debe subir la parrilla y calentar hasta el punto de ebullición para realizar una digestión ácida es importante mantener la ebullición por 30 min contados a partir de que empieza a hervir. Trascurrido el tiempo se añade $20 \mathrm{ml}$ de $\mathrm{NaOH}$, ebullicionando por $30 \mathrm{~min}$, se debe desconectar el vaso del condensador, enfriar y filtrar por crisol Gooch conteniendo en una capa de lana de vidrio y previamente tarado posteriormente se debe lavar el vaso y el residuo de papel con $250 \mathrm{ml}$ de agua destilada caliente y $15 \mathrm{ml}$ de hexano. Finalmente, se coloca el crisol en la estufa Memmert UN 30 a $105^{\circ} \mathrm{C}$ por $24 \mathrm{hr}$ para luego enfriarlo en un desecador y pesar. Por último colocar el crisol en la mufla Lindberg Blue $\mathrm{M}$ a $550^{\circ} \mathrm{C}$ por media hora, transcurrido el tiempo se debe enfriar en el desecador, pesar y realizar los cálculos con la siguiente fórmula:

$$
\% \text { de fibra } \frac{p 1-p}{m} \times 100,[18]
$$

P1: masa del crisol más el residuo desecado en la estufa en g

P: masa del crisol más las cenizas después de la incineración en mufla en g;

y m: masa de la muestra seca y desengrasada tomada para la determinación en g. 


\subsubsection{Determinación de humedad la harina de papa China}

La mayoría de los métodos para la determinación del contenido de agua en los alimentos se basan en la medición de la pérdida de peso debido a la evaporación de agua por la temperatura de ebullición o cerca de ella. Por tanto, la determinación de humedad se realiza con un $1 \mathrm{~g}$ de muestra (previamente realizado el desmuestre) en vidrio de reloj, colocándolo en la estufa Memmert UN 30 a $1030^{\circ} \mathrm{C}$ por un lapso de 2 a $3 \mathrm{hr}$, se debe enfriar en un desecador hasta la temperatura ambiente y se pesarlo en la abalanza analítica. La determinación debe realizarse por duplicado y con el cálculo de la siguiente fórmula.

$$
S S \% \frac{(\mathrm{m} 2-\mathrm{m} 1)}{\mathrm{m} 1-\mathrm{m}} \times 100,[19]
$$

donde:

SS: sustancia seca en porcentaje en; masa. m: masa de la cápsula en g;

m1: masa de la cápsula con la muestra en g; y

m2: masa de la cápsula con la muestra después del calentamiento en g

$$
\% \text { Humedad }=100-\% S S[20] .
$$

\subsubsection{Análisis del extracto etéreo en el vegetal fresco y la harina de papa China}

Se pesa $1 \mathrm{~g}$ de muestra seca y se colocó en el dedal; cubriendo la muestra con una porción de algodón desengrasado, se coloca el dedal dentro del porta dedal; añadiendo $25 \mathrm{ml}$ de éter etílico o éter de petróleo (se puede usar también hexano) en un vaso previamente tarado. Se coloca el vaso en el aparato de Goldfish con la ayuda de la rosca. Posteriormente se levanta las parrillas hasta tocar el vaso y encender el equipo, asegurándose la circulación de agua en el refrigerante. Después se procede a la extracción durante $3 \mathrm{hr}$ mínimo. Al término del tiempo, se debe bajar la parrilla, retirando el anillo de la rosca y el vaso conteniendo con el hexano más las sustancias extraídas. Se aparta el porta dedal y el dedal dejándolo desecar en la estufa, se debe enfriar en el desecador. A continuación, se sitúa el tubo recuperador en el porta dedal y nuevamente se coloca el vaso con la ayuda de la rosca. Se levanta la parrilla y se procede a calentar nuevamente para destilar el solvente en su mayor parte, después se debe bajar la parrilla y retirar el vaso conteniendo el extracto etéreo o grasa bruta o cruda. Por último, se coloca el vaso en la estufa durante media hora, se retira de la estufa, ubicándolo en el desecador, enfriarlo, pesarlo. La determinación debe realizarse por duplicado y con el cálculo de la siguiente fórmula:

$$
\% \mathrm{G}(\% \mathrm{Ex} . \mathrm{E})=\frac{(\mathrm{P} 1-\mathrm{P})}{m} * 100,[21]
$$


donde:

\%G: grasa cruda o bruta en muestra seca expresado en porcentaje en; masa

P1: masa del vaso más la grasa cruda o bruta extraída en g;

P: masa del vaso de extracción vacío en g; y

m: masa de la muestra seca tomada para la determinación en g.

\subsubsection{Análisis de los aminoácidos en la harina de papa China}

La determinación de aminoácidos se realiza a través de la cromatografía de gases preparando la muestra con eluyentes y estándares que incluyen en el Kit de aminoácidos. Cada eluyente y reactivo está compuesto de varios solventes orgánicos como cloroformo $\left(\mathrm{CHCl}_{3}\right)$, ácido clorhídrico $(\mathrm{HCl})$ e hidróxido de sodio $(\mathrm{NaOH})$ entre otros. La muestra se prepara en microlitros, y la estabilización del equipo se lo realiza con el técnico de laboratorio. El equipo a utilizar es un cromatografo de gases de marca perkin Elmer, con su respectiva columna para aminoácidos de derivatización. Las condiciones de la columna vienen establecidas de fábrica por lo tanto el generador de hidrógeno y el flujo de los gases deben estar en correcto funcionamiento para evitar daños dentro del equipo.

Se procede a preparar la muestra homogenizando $10 \mathrm{~g}$ de harina con $45 \mathrm{ml}$ de agua destilada y con ayuda de un papel filtro se debe separar los sólidos. A continuación se procede a realizar la hidrólisis en ácido clorhídrico al 15

$\%$, después se afora la muestra en un balón de foro de $50 \mathrm{ml}$. Dentro de la preparación de la muestra se realiza una hidrólisis ácida que permita separar la fase proteica de la fase grasa de la muestra de estudio, y se procede a extraer la cantidad de $2 \mathrm{ml}$ de la fase orgánica con la finalidad de inyectar correctamente en el equipo. Después se procede a visualizar las alturas y áreas del aminograma del pico para realizar el cálculo en porcentaje de los aminoácidos detectados en la muestra [22].

\subsubsection{Análisis del potencial de hidrógeno en la harina de papa China}

Este parámetro se realiza mediante la normativa ecuatoriana NTE INEN 389 [23], los $10 \mathrm{~g}$ de muestra de la harina del tubérculo de papa China se coloca en un vaso de precipitación con $100 \mathrm{ml}$ de agua previamente destilada. $Y$ a través de la decantación se procede a separar la mezcla de la fase sólida. Se debe calibrar el peachimetro con la solución buffer e introducir los electrodos del potenciómetro en el vaso de la muestra sin que estos toquen las paredes de recipiente, las mediciones se realizan por duplicado. 


\subsubsection{Análisis de la acidez titulable en la harina de papa China}

Para determinar la acidez se realiza por duplicado mediante la norma técnica NTE INEN-ISO 750 en la cual se homogeniza $10 \mathrm{~g}$ en $50 \mathrm{ml}$ de agua y con la ayuda de papel filtro se separa la fase sólida. En un vaso de precipitación se colocan $10 \mathrm{ml}$ de la muestra con 3 gotas de fenolftaleína para realizar el proceso de titulación con la ayuda de hidróxido de sodio. Mientras el hidróxido de sodio se va mezclando con la solución gota a gota se debe agitar hasta que cambie a un color rosado. Una vez que sucede el cambio de color anotar la cantidad de hidróxido de sodio que se utiliza en la titulación. La acidez titulable se expresa en porcentaje de ácido oxálico presente en la muestra y se calcula mediante la siguiente ecuación.

$$
A=V * 2 * F,
$$

donde:

A: acidez titulable en porcentaje de ácido específico (\% ácido oxálico/100 g de muestra);

V: volumen de solución de $\mathrm{NaOH}$ 0,1 $\mathrm{N}$ empleado en la titulación (ml); y

F: factor de acidez del ácido oxálico [0, 045] (INEN 2013a).

\subsubsection{Análisis de los grados Brix en la harina de papa China}

Para efectuar la medición de este parámetro se desarrolla bajo la norma ISO 21732013 se prepara agua destilada para limpiar la superficie del prisma colocando 0,3 $\mathrm{ml}$ de agua sobre el prisma, consecutivamente se presiona la tecla Start y si la pantalla indica $0,0 \%$ no se necesitara realizar al ajuste a cero, después se retira el agua de la superficie del prisma con un pañuelo de papel que no suelte pelusa, y con una pipeta se coloca aproximadamente $0,3 \mathrm{ml}$ de muestra sobre la superficie del prisma, se presiona la tecla STAR, el valor de la medición permanecerá en la pantalla durante aproximadamente dos minutos.

\subsubsection{Análisis microbiológicos en la harina de papa China}

Se realiza el análisis por duplicado de Escherichia coli, coliformes totales, aerobios mesófilos, salmonella, mohos y levaduras, con diluciones de 10-3, en la siembra se utiliza una pipeta de vidrio previamente esterilizada por calor en el mechero de Bunsen y en cajas petri film para evitar la contaminación, ubicándolas en la estufa memmert durante tiempos prolongados, trascurrido esta etapa se realiza el conteo para verificar o descartar la presencia de microorganismos [24]. 


\section{Determinación de coliformes totales, E. coli, salmonella y aerobios mesó- filos}

La determinación de coliformes totales se realiza en placas Petri film que contienen un indicador rojo que colorea todas las colonias y la capa superior atrapa el gas producido por los coliformes en su fase exponencial. Se prepara disoluciones de 10-3 levantando el film superior y con una pipeta perpendicular a la placa, se coloca $1 \mathrm{ml}$ de la muestra en el film inferior. Se debe bajar el film superior, evitando que se formen burbujas y luego aplicar una presión ligera sobre la placa para que distribuya la muestra en el cultivo.

Incubar las placas a una temperatura de $32^{\circ} \mathrm{C}$ por $24 \mathrm{hr}$. Para la determinación de aerobios mesófilos, salmonella, $E$. coli se realiza el mismo procedimiento en el medio agar standard method un agente gelificante soluble con indicador de color rojo (TTC), que facilita la enumeración de las colonias. Se debe incubar las placas a una temperatura de $32^{\circ} \mathrm{C}$ por $48 \mathrm{hr}$.

\section{Determinación de mohos y levaduras}

Para el recuento de mohos y levaduras se realiza en cultivos listos para usar que contiene nutrientes de Sabhi. Se debe preparar disoluciones de 10-3 levantando el film superior y con una pipeta perpendicular a la placa, se coloca $1 \mathrm{ml}$ de la muestra en el film inferior. Se debe bajar el film superior, evitando que se formen burbujas y luego aplicar una presión ligera sobre la placa para que distribuya la muestra en el cultivo. Incubar las placas a una temperatura de $32^{\circ} \mathrm{C}$ por $48 \mathrm{hr}$. Las levaduras aparecen normalmente como colonias pequeñas verde-azuladas con bordes definidos y sin foco central. Los mohos suelen mostrarse como colonias grandes, de color variable, con bordes difusos y foco central [25].

\section{Resultados y Discusión}

En la Tabla 1, muestra los valores obtenidos del análisis proximal del vegetal fresco ( $C$. esculenta); los parámetros evaluados son: humedad, cenizas, fibra, proteína y grasa.

En el parámetro humedad el tubérculo contiene un porcentaje del 72,01 \pm 0,25\% estos valores son similares a los reportados por Bradbury y Hollowoway (1988), con un valor de 69,01\%. Además, la Organización de Naciones Unidas para la Agricultura y la Alimentación (1997), reporta el valor nutricional de la papa China con un valor del 68,3\% de humedad [26].

En cuanto a las cenizas se obtiene el 1,20 $\pm 0,15 \%$ siendo superior a lo que reporta Bradbury y Hollowoway (1988), quien obtiene el 0,87\% para esta variable [27]. Por otra 


\section{Table 1}

Los valores obtenidos del análisis proximal de la hortaliza fresca (C. esculenta); los parámetros evaluados son: humedad, ceniza, fibra, proteína y grasa.

Parámetros
Humedad
Cenizas
Fibra
Proteína
Grasa

$$
\begin{aligned}
& \text { Resultados } \\
& 72,01 \pm 0,25 \\
& 1,20 \pm 0,15 \\
& 4,56 \pm 0,24 \\
& 2,42 \pm 0,12 \\
& 0,44 \pm 0.25
\end{aligned}
$$

parte, la fibra reportada en este tubérculo contiene el 4,56 $\pm 0,24 \%$; este valor es superior a lo que reporta Vanesa Zúñiga (2019) quien determinó este mismo parámetro con un valor de 3,52 $\pm 0,44 \%$ [28]. Además, para la variable proteína presenta un valor de 2,42 $\pm 0,12 \%$ y los resultados de los análisis químicos realizados por Andrés Gonzalez (2019) reporta un valor similar de 2,3\% [29]. Y de acuerdo con la (FAO, 1997), reporta un valor nutricional para la proteína de 1,4\% para esta especie. No obstante, también este valor es menor con lo que reporta (Corpei, 2013), quienes señalan el contenido de proteína con un valor de 1,7\% [8].

En cuanto, al contenido de extracto etéreo $(0,44 \pm 0,25 \%)$ en el tubérculo fue superior a lo que reporta Vanesa Zúñiga (2019), quien obtiene un valor de 0,36 $\pm 0,24 \%$. Mientras Palomino et al. reporta un porcentual similar 0,41\% para la harina de papa China [30]. Sin embargo, (Lisbeth Oñate, 2018) también obtiene un valor inferior de 0,34\% para la (C. esculenta Schott) de la variedad blanca. Un alto contenido de lípidos puede afectar a las propiedades funcionales y provocar la rancidez de la grasa presente en el almidón.

Cabe mencionar que las características químicas que poseen los distintas variedades de tubérculos dependen en gran medida de las propiedades del suelo donde fueron cultivados y cosechados así como de factores ambientales como la sequía y falta de nutriente [31].

\section{Table 2}

Los valores obtenidos del análisis proximal de la harina de papa China (C. esculenta); los parámetros evaluados son: humedad, cenizas, fibra, proteína y grasa.

Parámetros
Humedad
Cenizas
Fibra
Proteína
Grasa

Resultados
$9,01 \pm 0,21$
$2,24 \pm 0,15$
$4,25 \pm 0,26$
$2,02 \pm 0,02$
$0,43 \pm 0.15$


Se determina el análisis proximal del proceso de la harina de papa China y la Tabla 1 muestra sus resultados. El contenido del porcentaje de humedad de la harina presente es de (9,01\%), este valor concuerda con Aboubakar et al. (2008), que señala un rango de humedad entre $8,2 \%$ y $9,6 \%$ de seis variedades de papa China [32]. Además, estos valores son inferiores a lo reportado por Palomino et al. que también obtuvo resultados de 9,04\% [30]. El contenido de humedad puede estar relacionado con el almacenamiento y las condiciones de deshidratación del vegetal fresco, pues el exceso de humedad al $14 \%$ en harinas y almidones es susceptible a la contaminación con microorganismos. En cuanto al contenido de cenizas se reporta $(2,24 \pm)$ estos valores son inferiores a lo reportado por Aldaz, W (2011), obteniendo valores de 3,61\% de cenizas según, Palomino [30] manifiesta el 2,64\% para las harina de papa China [33].

En cuanto a la fibra dietética se obtuvo resultados de $(4,25 \pm 0,26)$, estos datos son superiores con lo que reporta (Wilian, C, 2018) hallando un valor de 1,46\% [27] y según Aldaz, W (2011) también reporta un valor de 0,82\% de fibra [33] datos inferiores a nuestra investigación. La fibra dietaría cuenta con varias propiedades funcionales como facilitar funciones alimenticias, procesos de reabsorción de compuestos no deseables como el colesterol, reducir tiempo de tránsito intestinal, aumenta la estabilidad de alimentos modificando su estructura, densidad y textura, así como la formación de gel en los alimentos y la capacidad de espesamiento en éstos, y las variedades que existen con respecto a la papa China podría explicar este resultado [34]. Ahora bien, en cuanto al contenido de proteína presente en la harina es del 2,02 $\pm 0,02 \%$ valor ligeramente superior a los reportados por Ríos (2014) entre 1,62\% y 2,00\% para tres variedades de papa China. Mientras que Torres et al. (2013) obtuvieron 1,5\% para la variedad blanca y 0,57\% para la morada. Además, (Alberto, T. Piedad, C. \& Marlene, L, 2013) reportó valores entre $2,9 \%$ y $4,9 \%$ para diferentes variedades de papa China [32].

Sin embargo, esta variable reside en una gran importancia biológica en su gran versatilidad funcional para la reparación de tejidos, la oxigenación del organismo y el favorable funcionamiento del sistema inmunológico [35].

En contenido de grasa presente en la harina $(0,43 \pm 0.15)$, valor superior a lo que reporta Oñate (2018), entre 0,34 $\pm 0,65 \%$ para la (C. esculenta Schott) de la variedad blanca. Mientras que Aboubakar et al. (2008) reportaron un contenido de lípidos entre 0,30\% y 1,17\% para seis variedades de papa China [32]. Un alto valor de lípidos presentes en los alimentos ocasiona la formación de peróxidos y la rancidez de la grasa que se encuentre en el mismo.

Además, en muchos cultivos de tubérculos el contenido de grasa es muy bajo, la cual está compuesta principalmente por los lípidos de la membrana celular que es variable entre los cultivares.

La Tabla 3 se observa el análisis físico obtenido de la harina de papa China previamente deshidratada. Respecto al perfil del potencial de hidrógeno presenta un $\mathrm{pH}$ 
Table 3

Los valores obtenidos del análisis físico la harina de la papa China (C. esculenta); los parámetros evaluados son: acidez, potencia de hidrógeno, y sólidos solubles totales.

\begin{tabular}{|l|l}
\hline Parámetros & Resultados \\
\hline Potencial de hidrógeno & $6,66 \pm 0,10$ \\
\hline Acidez & $2,24 \pm 0,00$ \\
\hline Grados Brix & $2 \pm 0,50$
\end{tabular}

de 6,66 \pm 0,10 encontrándose dentro de valores ácidos en la escala de $\mathrm{pH}$. Este valor obtenido es inferior a lo que reporta (Jesús, R. José R \& Emmanuel R 2011) con un valor de $6.78 \pm 0.00$. Además, Egan et al. (1981) menciona que el pH de las harinas oscila entre 6 y 6,8 corroborando con los datos obtenidos en este estudio [36], que pueden indicar un bajo contenido de ácidos orgánicos y alta concentración de almidones en la harina [37]. Sin embargo, el parámetro acidez es de 2,24 $\pm 0,00$ siendo un dato superior a lo que reporta (Lisbeth O, 2018, pág. 35) con un valor de 0,07 $\pm 0,01$ de acidez titulable (\% ácido oxálico).

Para el parámetro grados Brix se obtiene un valor de $2 \pm 0,50$ no obstante, es menor al presentado por (Lisbeth O, 2018, pág. 35) que obtuvo el 3,15 $\pm 0,08$. Este método determina la concentración de sacarosa de las frutas y hortalizas [2] que a través de los procesos fisiológicos que sufren los productos cosechados se trasforma el almidón presente en la papa China en los respectivos azúcares que los contiene. Por otra parte, las diferencias en la composición física encontradas en la presente investigación con otros cultivares de malanga ( $C$. esculenta $L$.) dependen de características extrínsecas e intrínsecas de la zona donde se cultive este tipo de especie, la temporada del cultivo y del grado de madurez del tubérculo que presente a la hora de la cosecha.

La Tabla 4 presenta los valores nutricionales de la harina de papa China expresados en porcentajes. La metionina es el valor más alto de $21,15 \%$. Estos datos concuerdan con lo reportado por (Laura, M \& Julia, H 2018), quienes mencionan en su estudio de aminoácidos fue alto, principalmente en los azufrados como la metionina para la harina del tubérculo de Malanga C. esculenta L. Schott [38]. Por lo que, (Jonathan G) establece que la metionina participa activamente en la descomposición de grasas y permite reducir el colesterol en la sangre. Ayuda a prevenir trastornos del cabello, piel y uñas.

Es antioxidante y participa en la síntesis de ARN y ADN [39]. Lo mismo sucede con el contenido de Alanina de un valor del 10,43\% y este resultado concuerda con (Laura, M \& Julia, H 2018), quienes reportan el $5,03 \pm 0,80 \%$ y además del ácido aspártico con un valor de $8,88 \%$ que se encuentran dentro del grupo de los aminoácidos no esenciales que nuestro organismo es capaz de sintetizarlos, y no necesitan ser consumidos diariamente a partir de la dieta [40]. 


\section{Table 4}

Los valores obtenidos del perfil de aminoácidos esenciales de la harina de papa China (C. esculenta).

\begin{tabular}{|c|c|c|c|c|c|c|}
\hline $\begin{array}{l}\text { Component } \\
\text { Name }\end{array}$ & Significado & Time [min] & $\begin{array}{l}\text { Area } \\
{[\mu \mathrm{V} . \mathrm{s}]}\end{array}$ & $\begin{array}{l}\text { Height } \\
{[\mu \mathrm{V} . \mathrm{s}]}\end{array}$ & $\begin{array}{l}\text { Concentración } \\
\text { UMOL/L }\end{array}$ & Porcentaje \% \\
\hline ALA & Alanine & 1.43 & 3563.07 & 3107.03 & 307.76 & 10.43 \\
\hline GLY & Glycine & 1.53 & 833.66 & 972.76 & 176.79 & 5.99 \\
\hline ABA & $\begin{array}{l}\alpha- \\
\text { Aminobutyric } \\
\text { acid }\end{array}$ & 1.61 & 124.32 & 170.68 & 8.46 & 0.29 \\
\hline BAIB & & 1.77 & 586.33 & 330.45 & 114.58 & 3.88 \\
\hline ISTD & & 1.85 & 1530.30 & 1490.53 & -ー-- & 0.00 \\
\hline LEU & Leucine & 1.89 & 121.84 & 91.52 & 8.25 & 0.28 \\
\hline AILE & $\begin{array}{l}\text { Allo- } \\
\text { Isoleucine }\end{array}$ & 1.93 & 1701.15 & 1419.93 & 238.28 & 8.08 \\
\hline THR & Threonine & 2.27 & 1459.97 & 1896.17 & 218.44 & 7.41 \\
\hline SER & Serine & 2.30 & 695.14 & 901.53 & 106.83 & 3.62 \\
\hline ASN & Asparagine & 2.39 & 4187.78 & 5388.13 & 0.00 & $2 e-05$ \\
\hline ASP & Aspartic acid & 2.88 & 1616.00 & 1027.89 & 261.89 & 8.88 \\
\hline MET & Methionine & 2.91 & 1509.23 & 4685.27 & 623.96 & 21.15 \\
\hline GLU & Glutamic acid & 3.24 & 862.35 & 632.74 & 198.64 & 6.73 \\
\hline PHE & Phenylalanine & 3.26 & 1196.02 & 1148.36 & 88.06 & 2.99 \\
\hline AAA & $\begin{array}{l}\alpha- \\
\text { Aminoaddipic } \\
\text { acid }\end{array}$ & 3.40 & 1518.42 & 1259.83 & 412.17 & 13.97 \\
\hline LIS & Lysine & 4.33 & 835.48 & 766.26 & 91.57 & 3.10 \\
\hline HIS & Histidine & 4.71 & 888.14 & 520.82 & 107.78 & 3.65 \\
\hline \multirow[t]{2}{*}{ TYR } & Tyrosine & 4.94 & 96.07 & 110.47 & -13.66 & -0.46 \\
\hline & & & 26925.28 & 25920.37 & 2949.82 & 100.00 \\
\hline
\end{tabular}

Asimismo, la Isoleucina reporta un valor del 8,08\% siendo necesario para la formación de hemoglobina, estabilizar y regular el azúcar en la sangre. Además, de ayudar en la curación del tejido muscular, piel y huesos [41]. Por otra parte, en cuanto al aminoácido Treonina el valor es de 7,41\% este valor es inferior a lo que reporta (Laura, M \& Julia, H 2018) $3,51 \pm 0,52$ cuya función es ayudar a mantener la cantidad adecuada de proteínas del cuerpo, la formación de colágeno, elastina y prevenir la acumulación de grasa en el hígado [41]

De la misma forma, los valores más bajos que presento la harina de este tubérculo es la Histidina con 3,65\% aminoácido utilizado en el tratamiento de alergias, ulceras y anemia. Además, de ser esencial para el mantenimiento de mielina que protegen las células nerviosas e importantes para la producción de glóbulos rojos y blancos en la sangre, preservando al organismo de los daños por radiación, reducir la presión arterial y eliminación de metales pesados del cuerpo. Si bien es cierto, la lisina que presenta 
esta investigación también es reducido 3,10\% es un aminoácido esencial que garantiza la absorción adecuada de calcio y mantiene un equilibrio adecuado de nitrógeno en adultos.

Al ser un aminoácido esencial, la dieta debe proveer la cantidad adecuada, ya que su deficiencia puede producir: anemia, pérdida de peso, inapetencia, retraso en el crecimiento, caída del cabello, falta de energía e irritabilidad [42].

\section{Table 5}

Los resultados obtenidos del análisis microbiológico de la harina papa China (C. esculenta); los parámetros evaluados son: Aerobios Mesófilos, E. Coli, Coliformes, Salmonella, mohos y levaduras.

\begin{tabular}{l|l|l|l|} 
Microorganismo & $\begin{array}{l}\text { Unidades formadoras } \\
\text { de colonias (UFC) }\end{array}$ & $\begin{array}{l}\text { Normativa (INEN, } \\
\mathbf{6 1 6 : 2 0 0 6} \text { ) }\end{array}$ & Unidad \\
\hline Aerobios Mesófilos & 76 & 100000 & ufc/g \\
\hline Escherichia coli & 3 & 0 & $\mathrm{ufc} / \mathrm{g}$ \\
\hline Coliformes & 3 & 100 & $\mathrm{ufc} / \mathrm{g}$ \\
\hline Salmonella & AUSENCIA & 0 & $\mathrm{ufc} / 25 \mathrm{~g}$ \\
\hline Mohosy levaduras & 1 & 500 & $\mathrm{ufc} / \mathrm{g}$
\end{tabular}

La Tabla 5 representa el análisis microbiológico de la harina de papa China, los aerobios mesófilos se reportan 76 ufc/g, encontrándose dentro de los parámetros que establece la normativa de un límite máximo de 100000 ufc/g de acuerdo al método de ensayo NTE INEN 1529-5. Por otra parte la presencia de coliformes es de 3 ufc/g, parámetro que se encuentra dentro de la norma permitida NTE INEN 1529-7 que estable un límite máximo de 100 ufc/g. Además, su valor es inferior a los resultados obtenidos por (Flavio, A \& Marcelo, G, 2011) que obtuvo 95 ufc/g [33]. Y en cuanto a la presencia de mohos y levaduras su valor es de $1 \mathrm{ufc} / \mathrm{g}$, esta cantidad se encuentra dentro de los parámetros que establece la normativa (INEN, 616:2006) que reporta un resultado de 500 ufc/g como límite máximo, bajo el método de ensayo NTE INEN 1529-10 [43].

En E. coli 3 ufc/g y Salmonella presenta ausencia de este microorganismo. Sin embargo, comparando estos resultados con los valores de la norma (INEN, 616:2006) observamos que tanto las bacterias totales, coliformes totales, E. coli mohos y levaduras se encuentran dentro de los valores establecidos por la normativa ecuatoriana.

\section{Conclusiones}

Los valores nutricionales reportados en la harina de papa China de la variedad blanca radica principalmente en su contenido de los aminoácidos esenciales; Metionina con un valor de 21,15\%, Alanina 10,43\%, Isoleucina 8,08\%, Treonina 7,41\%, Histidina 3,65\% y lisina con un valor de $3,10 \%$. Por lo que, puede ser utilizado como alimento funcional o ser trasformado mediante la utilización de distintas técnicas siendo así un importante 
recurso fitogenético para mejorar la calidad nutricional de los alimentos, la productividad, la sanidad alimentaria, así como la protección y prevención de enfermedades en zonas del ecuador con altos niveles de desnutrición.

El análisis proximal del vegetal fresco contiene el 72,01 \pm 0,25\% de humedad; 1,20 $\pm 0,15 \%$ de cenizas; $4,56 \pm 0,24$ de fibra; $2,42 \pm 0,12$ de proteína; $0,44 \pm 0,25 \%$ de grasa y el estudio proximal realizado en la harina presenta un porcentaje de humedad del 9,01 $\pm 0,21 \%$, cenizas $2,24 \pm 0,15$ fibra $4,25 \pm 0,26$ proteína $2,02 \pm 0,02$ y grasa $0,43 \pm 0.15$. De este modo, la deshidratación que se realiza en este tipo de alimento presenta cambios relativamente bajos que no influyen en los nutrientes de la harina ya procesada.

Se determinó los análisis microbiológicos de la harina de papa China para aerobios mesófilos 76 UFC, escherichia coli 3 UFC, Coliformes 3 UFC, Salmonella ausencia, mohos y levaduras 1 UFC. Concluyendo que se encuentran dentro de los parámetros establecidos en harinas de trigo con la norma INEN 616:2006 considerándola un producto inocuo que cumple que con la normativa microbiológica vigente siendo apta para el consumo humano y consecutivamente puede ser empleada en industria alimentaria. Esto nos permite impulsar al consumo de la harina de papa China de la variedad blanca.

\section{Agradecimientos}

Los autores agradecen a la BQF Alicia Zabala en el desarrollo, asesoría técnica en el laboratorio de bromatología animal de la facultad de ciencias pecuarias de la Espoch y el reporte de los resultados de cada una de las variables evaluadas en la investigación.

\section{References}

[1] Ararat Orozco M, Orozco MCA, Garcés CLS, Rivera CH. Valoraciones agronómicas y de rendimiento en la cosecha de "papa china" (Colocasia esculenta L.) en el trópico húmedo colombiano. Rev Investig Agrar Ambient. 1 de julio de 2014;5(2):169-80.

[2] 1112.pdf [Internet]. [citado 6 de enero de 2020]. Disponible en: http://repositorio.ug. edu.ec/bitstream/redug/3664/1/1112.pdf

[3] Barrera AFL. Propagación asexual bajo cuatro niveles de fertilización orgánica”. :120.

[4] a-i6881s.pdf [Internet]. [citado 7 de enero de 2020]. Disponible en: http://www.fao. org/3/a-i6881s.pdf

[5] 201_01WOCaicedo.pdf [Internet]. [citado 9 de enero de 2020]. Disponible en: http: //www.iip.co.cu/RCPP/201/201_01WOCaicedo.pdf 
[6] La papa, nutrición y alimentación - Año Internacional de la Papa 2008 [Internet]. [citado 6 de enero de 2020]. Disponible en: http://www.fao.org/potato2008/es/lapapa/hojas.html

[7] Martínez Augustin O, Martínez de Victoria E. Proteínas y péptidos en nutrición enteral. Nutr Hosp. mayo de 2006;21:01-14.

[8] Torres HJT. Sustitución parcial de harina de trigo (triticum aestivum I) por harina de papa china (colocasia esculenta) para la elaboración de galletas de dulce.:84.

[9] Greenfield H, Southgate DAT, Organització de les Nacions Unides per a l'Agricultura i l’Alimentació. Datos de composición de alimentos: obtención, gestión y utilización. Roma: Organización de las Naciones Unidas para la Agricultura y la Alimentación; 2006.

[10] Oj C-Q, Ga H-H, Jm R-T. Trastornos relacionados con el gluten: panorama actual. Med Interna México. 2017;16.

[11] LORSA.pdf [Internet]. [citado 7 de enero de 2020]. Disponible en: https://www. soberaniaalimentaria.gob.ec/pacha/wp-content/uploads/2011/04/LORSA.pdf

[12] Da Silva CA, Global Agro-Industries Forum, FAO, editores. Agroindustrias para el desarrollo: resultado del Foro Mundial sobre Agroindustrias. Rome: FAO; 2013. 306 p.

[13] Varela-Benavides I, Montero-Carmona W, Varela-Benavides I, Montero-Carmona W. Detección del virus del mosaico del tiquizque en aráceas comestibles mediante qPCR. Rev Tecnol En Marcha. junio de 2017;30(2):97-104.

[14] a0843s01.pdf [Internet]. [citado 8 de enero de 2020]. Disponible en: http://www.fao. org/3/a0843s/a0843s01.pdf

[15] sm2010-vp01b.pdf [Internet]. [citado 15 de junio de 2020]. Disponible en: https:// www.cenam.mx/sm2010/info/pviernes/sm2010-vp01b.pdf

[16] A173_es.pdf [Internet]. [citado 8 de enero de 2020]. Disponible en: https://www. itwreagents.com/download_file/brochures/A173/es/A173_es.pdf

[17] Determinacion cenizas metodo gravimetrico.pdf [Internet]. [citado 8 de enero de 2020]. Disponible en: http://www.idal.cl/sgcidal/images/stories/Procedimientos/ Laboratorio/Determinacion\{\%\}20cenizas\{\%\}20metodo\{\%\}20gravimetrico.pdf

[18] Manual de tecnicas para laboratorio de nutricion de peces y crustaceos [Internet]. [citado 8 de enero de 2020]. Disponible en: http://www.fao.org/3/AB489S/ AB489S03.htm

[19] Martínez EMG, Segovia IF. Determinación de la humedad de un alimento por un método gravimétrico indirecto por desecación. En 2012.

[20] Determinación de humedad [Internet]. [citado 8 de enero de 2020]. Disponible en: http://lqa2014.blogspot.com/p/informe-n-1-determinacion-de-humedad-1.html

[21] Manual de prácticos en alimentos.pdf [Internet]. [citado 8 de enero de 2020]. Disponible en: http://www.etpcba.com.ar/ 
DocumentosDconsulta/ALIMENTOS-Procesos\{\%\}20y\{\%]20qu\{\%\}c3\{\%\}8dmica/ manual\{\%\}20de\{\%\}20pr\{\%\}c3\{\%\}a1cticos\{\%\}20en\{\%\}20alimentos.pdf

[22] Novo JVJ, Díaz MNA, Ruiz JAB. 11. Separación de aminoácidos por cromatografía en capa fina y detección mediante reacción con ninhidrina.:11.

[23] Instituto Ecuatoriano de Normalización (INEN). NTE INEN 0389: Conservas vegetales. Determinación de la concentración del ión hidrógeno $(\mathrm{pH})$ [Internet]. 1986 [citado 9 de enero de 2020]. 7 p. Disponible en: http://archive.org/details/ec.nte. 0389.1986

[24] ManuallntegradoNormativMétodsDetecciónyMedOrganmsMicrobiol.pdf [Internet]. [citado 8 de enero de 2020]. Disponible en: https://www.cenam.mx/publicaciones/gratuitas/ descarga/pdf/ManuallntegradoNormativM\{\%\}C3\{\%\}A9todsDetecci\{\%\}C3\{\%\}B3nyMedOrganmsMicrobiol.pdf

[25] Hongos y Levaduras [Internet]. SIA. [citado 15 de junio de 2020]. Disponible en: http://siaasesoria.com.mx/productos/hongos-y-levaduras/

[26] Trabajo de Titulación.pdf [Internet]. [citado 14 de enero de 2020]. Disponible en: https://dspace.ucuenca.edu.ec/bitstream/123456789/32116/2/ Trabajo\{\%\}20de\{\%\}20Titulaci\{\%\}C3\{\%\}B3n.pdf

[27] Caicedo W. tubérculos de papa china (colocasia esculenta (I,) schott) como una fuente energética tropical para alimentar cerdos. una reseña corta sobre las características de la composición química y de los factores antinutricionales. 26 de junio de 2018;

[28] Unach-ec-ing.agro-ind-2019-0004.pdf [internet]. [citado 14 de enero de 2020]. disponible en: http://dspace.unach.edu.ec/bitstream/51000/5485/1/unach-ec-ing. agro-ind-2019-0004.pdf

[29] Ortiz E, Elizabeth G. Comportamiento térmico de la Colocasia esculenta (Papa china). 2019 [citado 14 de enero de 2020]; Disponible en: http://dspace.uazuay.edu.ec/ handle/datos/8698

[30] Palomino C, Molina Y, Pérez E. Physical and chemical characterization of flour and starches of tubers of Colocasia esculenta (L.) Schott and Xanthosoma sagittifolium (L.) Schott. Rev Fac Agron. 1 de enero de 2010;36:58-66.

[31] Onwueme IC. Taro cultivation in Asia and the Pacific. En 1999.

[32] Zúñiga LEO. Trabajo de Titulación, Modalidad Proyecto de Investigación, previa la obtención del Título de Ingeniero en Alimentos, otorgado por la Universidad Técnica de Ambato, a través de la Facultad de Ciencia e Ingeniería en Alimentos. :72.

[33] 075.pdf [Internet]. [citado 14 de enero de 2020]. Disponible en: http://www.dspace. ueb.edu.ec/bitstream/123456789/1188/1/075.pdf

[34] Fibra alimentaria - Fundación Hipercolesterolemia Familiar [Internet]. [citado 14 de enero de 2020]. Disponible en: https://www.colesterolfamiliar.org/habitos-de- vidasaludables/dieta-y-nutricion/fibra-alimentaria/ 
[35] Hernández DAQ. La importancia de las proteínas en la alimentación diaria [Internet]. 2017 [citado 14 de enero de 2020]. Disponible en: https://www.cubahora.cu/blogs/ consultas-medicas/la-importancia-de-las-proteinas-en-la-diaria-alimentacion

[36] AL 707.pdf [Internet]. [citado 19 de enero de 2020]. Disponible en: https://repositorio. uta.edu.ec/jspui/bitstream/123456789/30002/1/AL[\%]20707.pdf

[37] Rodriguez-Miranda J, Rivadeneyra-Rodríguez J, Ramírez-Rivera EJ, Juárez- Barrientos J, Herrera-Torres E, O. N-C, et al. Caracterización fisicoquímica, funcional y contenido fenólico de harina de malanga (Colocasia esculenta) cultivada en la región de Tuxtepec, Oaxaca, México. Cienc Mar. 1 de enero de 2011;15:37-47.

[38] Madrigal-Ambriz LV, Hernández-Madrigal JV, Carranco ME. Caracterización física y nutricional de harina del tubérculo de "Malanga" (Colocasia esculenta L. Schott) de Actopan, Veracruz, México.:9.

[39] Tabla de aminoácidos: funciones, tipos y características [Internet]. 2017 [citado 23 de enero de 2020]. Disponible en: https://psicologiaymente.com/neurociencias/ tabla-de-aminoacidos

[40] Alanina, aminoácido no esencial: funciones y beneficios [Internet]. Natursan. 2013 [citado 23 de enero de 2020]. Disponible en: https://www.natursan.net/ alanina-aminoacido-no-esencial/

[41] Lista de aminoácidos y sus funciones.pdf [Internet]. [citado 23 de enero de 2020]. Disponible en: https://rinconeducativo. com/datos/Cosmetolog\{\%\}C3\{\%\}ADa/Documentaci\{\%\}C3\{\%\}B3n/ lista\{\%]20de\{\%\}20amino\{\%\}C3\{\%\}A1cidos\{\%\}20y\{\%\}20sus\{\%\}20funciones.pdf

[42] Funciones y propiedades de los aminoácidos (alfabético desde A hasta L) [Internet]. Zonadiet. [citado 23 de enero de 2020]. Disponible en: https://www.zonadiet.com/ nutricion/aminoacidos2.htm

[43] NTE INEN 0616: Harina de trigo. Requisitos [Internet]. studylib.es. [citado 14 de enero de 2020]. Disponible en: https://studylib.es/doc/5531663/ nte-inen-0616--harina-de-trigo.-requisitos 